\title{
Areas of normal pulmonary parenchyma on HRCT exhibit increased FDG PET signal in IPF patients
}

\author{
Thida Win • Benjamin A. Thomas • Tryphon Lambrou • Brian F. Hutton • \\ Nicholas J. Screaton • Joanna C. Porter • Toby M. Maher • Raymondo Endozo • \\ Robert I. Shortman • Asim Afaq • Pauline Lukey • Peter J. Ell • Ashley M. Groves
}

Received: 29 January 2013 / Accepted: 7 July 2013 / Published online: 14 August 2013

(C) The Author(s) 2013. This article is published with open access at Springerlink.com

\begin{abstract}
Purpose Patients with idiopathic pulmonary fibrosis (IPF) show increased PET signal at sites of morphological abnormality on high-resolution computed tomography (HRCT). The purpose of this investigation was to investigate the PET signal at sites of normal-appearing lung on HRCT in IPF. Methods Consecutive IPF patients ( 22 men, 3 women) were prospectively recruited. The patients underwent ${ }^{18} \mathrm{~F}-\mathrm{FDG}$ $\mathrm{PET} / \mathrm{HRCT}$. The pulmonary imaging findings in the IPF patients were compared to the findings in a control population. Pulmonary uptake of ${ }^{18}$ F-FDG (mean SUV) was quantified at sites of morphologically normal parenchyma on HRCT. SUVs were also corrected for tissue fraction (TF). The mean SUV in IPF patients was compared with that in 25 controls (patients with lymphoma in remission or suspected paraneoplastic syndrome with normal PET/CT appearances).
\end{abstract}

T. Win

Respiratory Medicine, Lister Hospital, Stevenage, UK

B. A. Thomas - T. Lambrou • B. F. Hutton • R. Endozo • R. I. Shortman • A. Afaq • P. J. Ell • A. M. Groves $(\bowtie)$ Institute of Nuclear Medicine, University College Hospital, University College London, 5th Floor, 235 Euston Road, London NW1 2BU, UK

e-mail: ashleygroves@nhs.net

N. J. Screaton

Radiology Department, Papworth Hospital, Papworth Everard, UK

J. C. Porter

Centre for Respiratory Diseases, University College Hospital, University College London, Euston Rd, London, UK

T. M. Maher

Interstitial Lung Disease Unit, Royal Brompton Hospital,

London, UK

P. Lukey

Fibrosis DPU, Research and Development, GSK, Stevenage, UK
Results The pulmonary SUV (mean $\pm \mathrm{SD}$ ) uncorrected for $\mathrm{TF}$ in the controls was $0.48 \pm 0.14$ and $0.78 \pm 0.24$ taken from normal lung regions in IPF patients $(p<0.001)$. The TFcorrected mean SUV in the controls was $2.24 \pm 0.29$ and $3.24 \pm 0.84$ in IPF patients $(p<0.001)$.

Conclusion IPF patients have increased pulmonary uptake of ${ }^{18} \mathrm{~F}-\mathrm{FDG}$ on PET in areas of lung with a normal morphological appearance on HRCT. This may have implications for determining disease mechanisms and treatment monitoring.

Keywords Idiopathic pulmonary fibrosis $\cdot \mathrm{PET} / \mathrm{CT}$

\section{Introduction}

Idiopathic pulmonary fibrosis (IPF) has an increasing incidence and a prognosis worse than many cancers. There remains no effective treatment. The current main role of imaging in IPF has been the use of high-resolution computed tomography (HRCT) both for diagnosis and monitoring. However, the impact of HRCT on mortality in IPF is limited [1-5]. There have been recent reports on the use of PET in IPF. Increased pulmonary PET signal with various PET tracers is now documented in IPF and other diffuse parenchymal pulmonary diseases (DPLD). These signal changes have been related to regions of lung parenchymal changes on HRCT [6-11]. However, to our knowledge, no studies have addressed metabolic functional changes in morphologically normal lung on HRCT in the setting of IPF. Identification of abnormal PET signal in IPF, undetectable on HRCT, could give an insight into the pathogenesis of the disease and may permit earlier detection of changes in disease activity. The latter could be important in disease monitoring. Sensitive methods of disease monitoring in IPF patients are urgently required, as a lack of early measurable endpoints is hampering drug development. 
Table 1 Clinical profiles of the 25 IPF patients

\begin{tabular}{|c|c|c|c|c|}
\hline \multirow[t]{2}{*}{ Patient } & \multirow[t]{2}{*}{ Sex } & \multirow{2}{*}{$\begin{array}{l}\text { Age } \\
\text { (years) }\end{array}$} & \multicolumn{2}{|c|}{ Pulmonary function tests ${ }^{\mathrm{a}}$} \\
\hline & & & $\begin{array}{l}\text { Forced vital } \\
\text { capacity }\end{array}$ & $\begin{array}{l}\text { Carbon monoxide } \\
\text { transfer factor }\end{array}$ \\
\hline 1 & M & 74 & 56 & 51 \\
\hline 2 & M & 56 & 99 & N/A \\
\hline 3 & M & 75 & 61 & 69 \\
\hline 4 & M & 72 & 41 & N/A \\
\hline 5 & M & 86 & 75 & 37 \\
\hline 6 & M & 73 & 98.9 & 68.6 \\
\hline 7 & M & 73 & 89 & 42 \\
\hline 8 & M & 71 & 101.1 & N/A \\
\hline 9 & M & 76 & 91 & 59 \\
\hline 10 & M & 74 & 70 & N/A \\
\hline 11 & M & 74 & 63 & 34 \\
\hline 12 & M & 81 & 67 & N/A \\
\hline 13 & M & 56 & 62 & 26 \\
\hline 14 & M & 66 & 70 & 40 \\
\hline 15 & M & 76 & 87 & 62 \\
\hline 16 & M & 82 & 119 & 74 \\
\hline 17 & F & 71 & 106 & 45 \\
\hline 18 & $\mathrm{M}$ & 72 & N/A & N/A \\
\hline 19 & $\mathrm{M}$ & 71 & N/A & N/A \\
\hline 20 & $\mathrm{~F}$ & 63 & 68.7 & 37.3 \\
\hline 21 & $\mathrm{M}$ & 77 & 86 & 56 \\
\hline 22 & $\mathrm{M}$ & 73 & 52 & N/A \\
\hline 23 & $\mathrm{~F}$ & 65 & 80 & 57 \\
\hline 24 & $\mathrm{M}$ & 77 & 101 & 62 \\
\hline 25 & $\mathrm{M}$ & 86 & 104 & 70 \\
\hline
\end{tabular}

N/A not applicable

${ }^{\text {a }}$ Pulmonary function test data are percentage predicted

For these reasons we measured the ${ }^{18} \mathrm{~F}$-FDG uptake using PET in regions of lung parenchyma with normal HRCT appearances in IPF patients, and compared the uptake to that in morphologically normal areas of lungs in patients without lung disease.

\section{Materials and methods}

Patients

\section{IPF patients}

Consecutive IPF patients (22 men, 3 women; age $72.8 \pm 7.5$ years, mean $\pm \mathrm{SD}$ ) were prospectively recruited for ${ }^{18} \mathrm{~F}$-FDG PET imaging. All patients underwent full clinical assessment including multidisciplinary team review, pulmonary function tests and HRCT evaluation. Infection and neoplasia were excluded on clinical and radiological grounds. The diagnosis of IPF was made on clinical/radiological grounds following multidisciplinary team review. The clinical profiles of the patients are summarized in Table 1 . The study was approved by the ethics board.

\section{Control subjects}

Due to the relatively high radiation exposure from ${ }^{18} \mathrm{~F}$-FDG PET/CT, normal volunteers were not used. Instead, two patient groups were used as a control population: 15 patients (7 men, 8 women; $63.9 \pm 11.4$ years) undergoing ${ }^{18} \mathrm{~F}-\mathrm{FDG}$ PET/CT referred with neurological conditions to exclude paraneoplastic syndrome (with negative findings) and 10 patients (5 men, 5 women; $59.1 \pm 10.4$ years) with extrathoracic lymphoma (1 Hodgkin's lymphoma and 9 non-Hodgkin's) in remission undergoing routine surveillance off treatment. HRCT was not performed in the control population. All these patients were consecutively recruited over 12 months. As the subjects in both groups were expected to have healthy lung parenchyma, the lymphoma and paraneoplasia subjects were combined into a single control group (62.16 \pm 10.3 years) for comparison with IPF patients.

\section{PET/CT acquisition}

All patients fasted for $6 \mathrm{~h}$. Images were acquired $1 \mathrm{~h}$ after injecting $200 \mathrm{MBq}$ of ${ }^{18} \mathrm{~F}$-FDG using a dedicated combined
Fig. 1 PET/CT images in a patient with IPF. A region of interest is drawn on normal lung parenchyma shown on the CT images (upper row). Normality was confirmed by an experienced ( $>10$ years) thoracic radiologist. Corresponding PET images (lower row) with the region of interest superimposed allow quantification of ${ }^{18} \mathrm{~F}-\mathrm{FDG}$ uptake

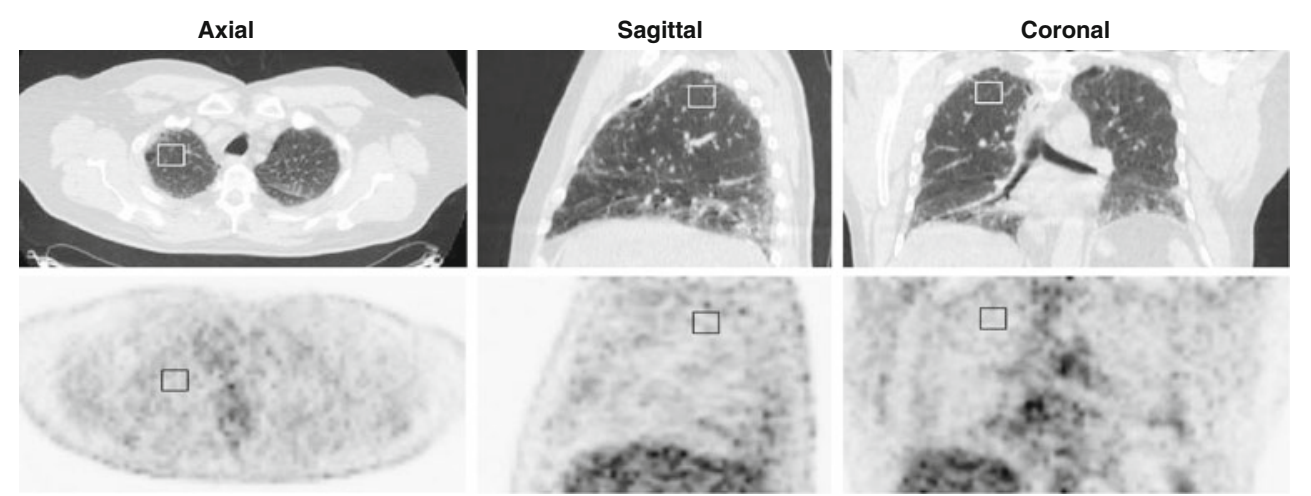


Mean Hounsfield Unit (HU)

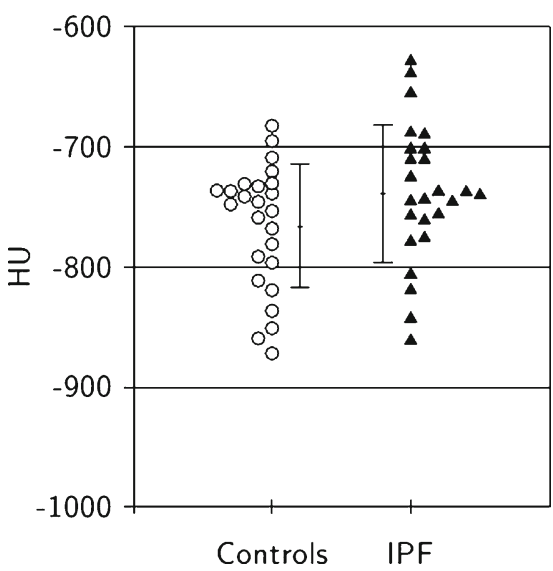

Fig. 2 Pulmonary parenchyma CT densities (Hounsfield units) in IPF and control subjects (error bars mean $\pm \mathrm{SD}$ )

PET/64-detector CT instrument (GE Healthcare Technology, Waukesha, WI). In total, three sequential imaging sequences of the thorax were performed whilst the patient remained supine on the table throughout. Firstly, a CT scan was performed for attenuation correction using $64 \times 3.75$ $\mathrm{mm}$ detectors, a pitch of 1.5 and a $5-\mathrm{mm}$ collimation $(140 \mathrm{kVp}$ and $80 \mathrm{~mA}$ in $0.8 \mathrm{~s}$ ). Maintaining the patient position, a whole-body ${ }^{18} \mathrm{~F}$-FDG PET emission scan was performed covering an area identical to that covered by the CT scan. All acquisitions were carried out in 2-D mode ( 8 min per bed position). Transaxial emission images of $3.27-\mathrm{mm}$ thickness (pixel size $3.9 \mathrm{~mm}$ ) were reconstructed using ordered subsets expectation maximization with two iterations and 28 subsets. The axial field of view was $148.75 \mathrm{~mm}$, resulting in 47 slices per bed position. Next, maintaining the patient position, a deep inspiratory HRCT scan was performed using $64 \times 1.25$-mm detectors, a pitch of 0.53 and $1.25-\mathrm{mm}$ collimation (120 kVp and $100 \mathrm{mAs}$ ).

Image analysis

\section{Observers}

Images were analysed by a physicist with $>3$ years' experience, a PET radiologist with $>5$ years' experience and a senior PET technologist with $>5$ years' experience in quantifying pulmonary PET images in IPF patients. All corresponding CT images were analysed for normality by a dedicated chest radiologist with a $>10$ years' special interest in HRCT and $>5$ years' experience at analysing CT and HRCT with PET scans in IPF patients.

\section{Image display and processing}

All images were loaded onto a proprietary workstation. All datasets underwent registration [12] to take into account respiratory mismatches between PET and CT studies as previously described [13, 14]. A single volume of interest (VOI) was placed in each lung in areas considered morphologically normal. Care was taken to identify normal regions of lung identifiable over several transverse slices, remote from major blood vessels and airways (see Fig. 1). In most subjects regions were defined in the middle lobe; in some subjects 'normal' regions could only be identified closer to the apex. Relatively small regions were defined so as to avoid spill-over from adjacent tissues. Care was also taken to select the central slices from the subvolume containing normal lung so as to minimize the effects of axial motion. In IPF subjects, normal regions corresponded to areas of minimal observed density as distinct from regions that were identifiable as having either ground glass or honeycomb patterns or more diffuse increases in density. Observers were requested to define VOIs with a target volume of $20 \mathrm{~cm}^{3}$. In subjects in whom a volume of this size was not identifiable, smaller VOIs were selected. The median (range) VOI in the control group was $21.23 \mathrm{~cm}^{3}\left(17.05-22.01 \mathrm{~cm}^{3}\right)$ and in the IPF group was $19.37 \mathrm{~cm}^{3}\left(15.31-22.01 \mathrm{~cm}^{3}\right)$. VOIs were then used on the coregistered PET studies to determine the mean SUV within each VOI. Analysis was performed on the datasets with and without correction for tissue fraction (TF) in order to account for the variable amount of air in different lung regions (both within individuals and across the subjects studied), as described previously [13, 14].

\section{Statistical analysis}

Control and patient groups were compared in terms of mean Hounsfield units and mean SUV, with and without TF correction, using two-tailed $t$ tests, assuming unequal variance. The intragroup variability, with and without TF correction, was also reported in terms of coefficient of variation $(\mathrm{CoV})$, calculated as the group SD divided by the group mean.

Table 2 CT densities (Hounsfield units) in pulmonary regions selected as normal on CT scans in IPF and control subjects

\begin{tabular}{|c|c|c|c|c|c|}
\hline \multirow[t]{2}{*}{ Group } & \multicolumn{2}{|c|}{ CT density (HU) } & \multirow[t]{2}{*}{$p$ value } & \multicolumn{2}{|c|}{$95 \%$ confidence interval } \\
\hline & Mean & $\mathrm{SD}$ & & Lower bound & Upper bound \\
\hline Control & -765.97 & 51.54 & 0.08 & -786.17 & -745.77 \\
\hline IPF & -738.64 & 57.60 & & -761.22 & -716.06 \\
\hline
\end{tabular}


Table 3 Uncorrected and TFcorrected mean SUVs using the normal part of IPF lungs in the comparison. The $p$ values reflect the significance of the difference between the control and IPF groups

\begin{tabular}{|c|c|c|c|c|c|c|}
\hline \multirow[t]{2}{*}{ TF correction } & \multirow[t]{2}{*}{ Group } & \multicolumn{2}{|l|}{ SUV } & \multirow[t]{2}{*}{$p$ value } & \multicolumn{2}{|c|}{$95 \%$ confidence interval } \\
\hline & & Mean & SD & & Lower bound & Upper bound \\
\hline \multirow[t]{2}{*}{ Uncorrected } & Control & 0.48 & 0.14 & 0.00000423 & 0.43 & 0.54 \\
\hline & IPF & 0.78 & 0.24 & & 0.69 & 0.88 \\
\hline \multirow[t]{2}{*}{ Corrected } & Control & 2.24 & 0.29 & 0.00000434 & 2.13 & 2.36 \\
\hline & IPF & 3.24 & 0.84 & & 2.91 & 3.57 \\
\hline
\end{tabular}

\section{Results}

No significant difference in SUV was found between the lymphoma and paraneoplasia groups. This was true both for uncorrected SUV (lymphoma group 0.45 \pm 0.13 ; paraneoplasia group $0.51 \pm 0.14$, mean $\pm \mathrm{SD} ; p=0.25)$ and TFcorrected mean SUV (lymphoma group 2.07 \pm 0.30 ; paraneoplasia group $2.27 \pm 0.31 ; p=0.11$ ). This justified the combination of the lymphoma and paraneoplasia groups into a single control group.

The pulmonary parenchyma CT densities (Hounsfield units) of the control groups and the IPF patients were not significantly different (Fig. 2 and Table 2; $p=0.08$ ), supporting the visual selection of normal regions in IPF patients as assessed by the HRCT expert reporter.

The difference in mean SUV between the combined control group and the IPF group were highly significant both for data uncorrected for TF $(0.48 \pm 0.14$ versus $0.78 \pm 0.24$; Table 3 and Fig. $3 \mathrm{a} ; p<0.00001)$ and for data corrected for TF (2.24 \pm 0.29 versus 3.24 \pm 0.84 ; Table 3 and Fig. 3b; $p<0.00001)$. The CoVs of the uncorrected data of the two groups were 0.29 for the combined control group and 0.31 for the IPF group. After TF correction, the CoVs reduced to 0.13 for the control group and 0.26 for the IPF subjects.

\section{Discussion}

These data show that in IPF there is increased pulmonary uptake of ${ }^{18}$ F-FDG on PET even in those areas with a normal morphological appearance of the lung parenchyma on HRCT. Our analysis of the CT data confirmed that the selected normal regions in IPF patients were appropriate, yielding Hounsfield unit values consistent with those of the normal group. This implies that PET may have a higher sensitivity in the detection of early pulmonary disease in IPF than HRCT. This has possible implications for understanding the in vivo pathogenesis of IPF and may be important in early disease detection and treatment monitoring. The pathogenesis of IPF remains poorly understood [1-4]. Our finding that there is diffuse hypermetabolism implies that the parenchyma is globally affected in IPF. How this can be reconciled with HRCT changes that predominate in the lower lobe and peripherally is uncertain, but it is recognized that the pathological changes in IPF are both spatially and temporally heterogeneous. The cell type accounting for the metabolic signal on FDG PET also remains unclear. The presence of diffusely raised pulmonary ${ }^{18} \mathrm{~F}$-FDG uptake, even in areas of normal parenchyma on HRCT, renders hypotheses suggesting that the PET signal in IPF patients is
Fig. 3 Mean pulmonary SUVs in IPF and control subjects (a uncorrected for $\mathrm{TF}$, $\mathbf{b}$ corrected for TF; error bars mean $\pm \mathrm{SD}$ ) a Mean SUV (Uncorrected)

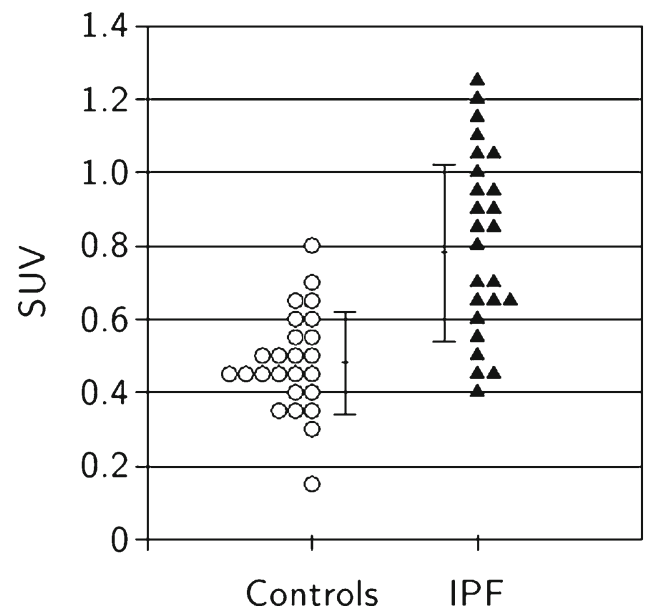

b

Mean SUV (TF-corrected)

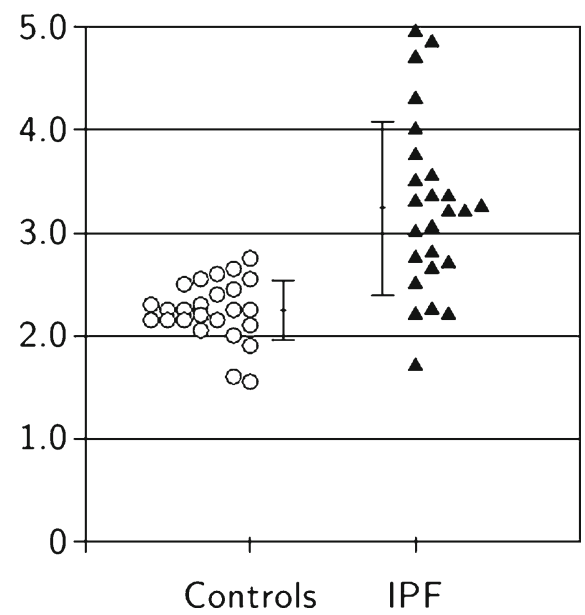


predominantly caused by colonization of polymorph infiltration secondary to infection in dilated airways less likely. Moreover, the fact that fibroblasts are generally sparse in normal lung [15] may suggest that this cell type is not responsible for the PET signal.

We present the first evidence in IPF patients that PET can detect abnormalities in the absence of HRCT lung parenchymal changes. This suggests that PET may be more sensitive than HRCT in detecting early disease, and in turn, this may allow early detection of therapeutic response. It has been shown $[16,17]$ that honeycombing and reticulation changes on HRCT are associated with microscopic changes of fibrosis (usual interstitial pneumonia pattern). Once the disease has reached this stage, it would be challenging to reverse the process. However, detecting earlier disease signals may help reveal upstream metabolic targets for switching off the fibrotic drive. In this respect, the role of TGF-beta and integrin metabolism is becoming increasingly recognized in IPF and thus there is potential for antagonists such as those against alpha $\mathrm{v}$ beta 6 ligands to modify the disease process [18].

In the development of such therapeutics for IPF, our PET findings may play a role by potentially offering early disease detection and monitoring of therapeutic response to treatment. There are no effective treatments and drug discovery trials are limited by the only current reliable endpoint of mortality. Since mortality is a relatively long endpoint (typically $>2$ years), this is a considerable barrier to therapeutic development [1-4]. Given that it has been shown that the pulmonary PET signal in IPF is stable over time [14], this would further support the development of PET as a surrogate endpoint for future clinical trials.

We have previously drawn attention to the importance of correcting for the variable air volume in the lung by performing TF correction [13]. In this study applying this correction increased the difference between the disease and control groups, while reducing intragroup variability. This suggests that TF correction may be important in maximizing sensitivity to detection of early disease.

The study limitations include the lack of data from normal volunteers. However, given the radiation exposure from ${ }^{18} \mathrm{~F}$ FDG PET/CT, there would be ethical concerns in acquiring data from healthy patients. Similarly, HRCT was not performed in the control population due to the associated increase in CT dose. This may have reduced the accuracy of VOI placement in the controls compared to that in the IPF patients who all had HRCT data available. The age and sex profile of the IPF patients make it difficult to find suitable control groups matched for age and sex. We therefore selected the 25 oldest subjects (male and female) from our two control cohorts. We found no significant differences in the results between the two patient subgroups used for controls. There was some heterogeneity in the patients with IPF, such as administered medications, which may have altered the pulmonary SUV measurements. However, a degree of heterogeneity is always present in IPF/DPLD populations and our population was relatively homogeneous compared to others. No respiratory gating was employed in the study and so there is the possibility of mismatch between the PET data (which is averaged over the respiratory cycle) and the short-acquisition CT study. The datasets were registered to minimize differences between the studies. Also relatively small regions were defined to accommodate the fact that SUV values represent average values for tissue extending beyond the defined regions as a result of respiratory motion.

\section{Conclusion}

IPF patients have increased pulmonary uptake of ${ }^{18} \mathrm{~F}$-FDG on PET at sites with normal morphological appearance on lung HRCT. These findings may have implications for the understanding of the pathogenesis of this disease and its treatment.

Acknowledgments This work was undertaken at UCLH/UCL, which received a proportion of the funding from the UK's Department of Health's NIHR Biomedical Research Centre's funding scheme.

Conflicts of interests We acknowledge a proportion of funding and input from GSK (CRT115549) Research and Development in Stevenage, UK, and the GSK CRAFT consortium.

Open Access This article is distributed under the terms of the Creative Commons Attribution License which permits any use, distribution, and reproduction in any medium, provided the original author(s) and the source are credited.

\section{References}

1. Coultas DB, Zumwalt RE, Black WC, Sobonya RE. The epidemiology of interstitial lung diseases. Am J Respir Crit Care Med. 1994;150:967-72.

2. Raghu G, Weycker D, Edelsberg J, Bradford WZ, Oster G. Incidence and prevalence of idiopathic pulmonary fibrosis. Am J Respir Crit Care Med. 2006;174:810-6.

3. Gross TJ, Hunninghake GW. Idiopathic pulmonary fibrosis. N Engl J Med. 2001;345:517-25.

4. Noth I, Martinez FJ. Recent advances in idiopathic pulmonary fibrosis. Chest. 2007;132:637-50.

5. Raghu G, Collard HR, Egan JJ, Martinez FJ, Behr J, Brown KK, et al. ATS/ERS/JRS/ALAT Committee on Idiopathic Pulmonary Fibrosis. An official ATS/ERS/JRS/ALAT statement: idiopathic pulmonary fibrosis: evidence-based guidelines for diagnosis and management. Am J Respir Crit Care Med. 2011;183:788-824.

6. Groves AM, Win T, Screaton NJ, Berovic M, Endozo R, Booth H, et al. Idiopathic pulmonary fibrosis and diffuse parenchymal lung disease: implications from initial experience with 18F-FDG-PET/ CT. J Nucl Med. 2009;50:538-45.

7. Umeda Y, Demura Y, Ishizaki T, Ameshima S, Miyamori I, Saito Y, et al. Dual-time-point (18)F-FDG PET imaging for diagnosis of disease type and disease activity in patients with idiopathic interstitial pneumonia. Eur J Nucl Med Mol Imaging. 2009;36:1121-30. 
8. Nusair S, Rubinstein R, Freedman NM, Amir G, Bogot NR, Izhar $\mathrm{U}$, et al. Positron emission tomography in interstitial lung disease. Respirology. 2007;12:843-7.

9. Meissner H, Soo Hoo GW, Khonsary SA, Mandelkern M, Brown CV, Santiago SM. Idiopathic pulmonary fibrosis: evaluation with positron emission tomography. Respiration. 2006;73:197-202.

10. Ambrosini V, Zompatori M, De Luca F, Antonia D, Allegri V, Nanni C, et al. 68Ga-DOTANOC PET/CT allows somatostatin receptor imaging in idiopathic pulmonary fibrosis: preliminary results. J Nucl Med. 2010;51:1950-5.

11. Win T, Screaton NJ, Porter JC, Endozo R, Kayani I, Dickson J, et al. Novel PET/CT imaging of diffuse parenchymal lung disease combining a labelled somatostatin receptor analogue and 18F-FDG. Mol Imaging. 2012;11:91-8.

12. Modat M, Ridgway GR, Taylor ZA, Lehmann M, Barnes J, Hawkes DJ, et al. Fast free-form deformation using graphics processing units. Comput Methods Prog Biomed. 2010;98:278-84.

13. Lambrou T, Groves AM, Erlandsson K, Win T, Screaton N, Porter $\mathrm{JC}$, et al. The importance of correction for tissue fraction effects in lung PET; preliminary investigation. Eur J Nucl Med Mol Imaging. 2011;38:2238-46.

14. Win T, Lambrou T, Hutton BF, Screaton N, Porter JC, Maher T, et al. 18F-Fluorodeoxyglucose positron emission tomography pulmonary imaging in idiopathic pulmonary fibrosis is reproducible: implications for future clinical trials. Eur J Nucl Med Mol Imaging. 2012;39:521-8

15. Hamid Q, Martin J, Shannon J. Physiological basis of respiratory disease. Hamilton, Ontario: BC Decker; 2005.

16. Flaherty KR, Toews GB, Travis WD, Colby TV, Kazerooni EA, Gross BH, et al. Clinical significance of histological classification of idiopathic interstitial pneumonia. Eur Respir J. 2002;19:275-83.

17. Katzenstein AL, Myers JL. Idiopathic pulmonary fibrosis: clinical relevance of pathologic classification. Am J Respir Crit Care Med. 1998;157:1301-15.

18. Goodwin A, Jenkins G. Role of integrin-mediated TGFbeta activation in the pathogenesis of pulmonary fibrosis. Biochem Soc Trans. 2009;37:849-54. 